\title{
УДК 622.276:004.896
}

\section{ГЛУБОКИЕ ИСКУССТВЕННЫЕ НЕЙРОННЫЕ СЕТИ ДЛЯ ПРОГНОЗА ЗНАЧЕНИЙ ДЕБИТОВ ДОБЫВАЮЩИХ СКВАЖИН}

\author{
Евсюткин Иван Викторович1, \\ ive1@tpu.ru
}

\author{
Марков Николай Григорьевич 1 , \\ markovng@tpu.ru \\ 1 Национальный исследовательский Томский политехнический университет,
Россия, 634050, г. Томск, пр. Ленина, 30.
}

\begin{abstract}
Актуальность исследования обусловлена необходимостью поддержки принятия решения спещиалистами добывающих предприятий нефртегазовой отрасли при управлении производством. Точное прогнозирование значений дебитов добывающих скважин позволяет определить такие технологические режимы работы фонда скважин и технологического оборудования, которые позволят достичь заданного объёма выпуска продукции. Существующие методы не всегда обеспечивают требуемый уровень точности при прогнозе значений дебитов скважин, что приводит к ошибкам при расчёте экономического эфрфекта при оценке рентабельности добывающих скважин и последующих поставок уелеводородного сырья, а также при учёте ограничений, накладываемых органами надзора за пользованием недрами.

Цель: разработать и предложить наиболее эфффективные модели глубоких искусственных нейронных сетей при прогнозе значений компонентов добычи углеводородного сырья - нефти, газа, жидкости (газового конденсата) и воды.

Объекты: технологические параметры дебитов добывающих скважин фонда нефтяных, газовых, нефтегазовых и нефртегазоконденсатных месторождений.

Методы: методы анализа больших объёмов технологических данных скважин, развиваемые в соответствии с концепцией «Big Data»; модели глубоких искусственных нейронных сетей; объектно-ориентированное программирование; методы оценки и статистического анализа результатов исследований эфрфективности глубоких искусственных нейронных сетей при прогнозе значений дебитов добывающих скважин.

Результаты. Разработана методика подготовки данных по дебитам скважин для обучения и тестирования глубоких искусственных нейронных сетей прямого распространения. Проведены исследования различных архитектур таких нейросетей при решении задач прогноза дебитов нефти, газа, жидкости (газового конденсата) и дебита воды. Выявлены наиболее эфрфективные архитектуры глубоких нейросетей прямого распространения. Такие нейронные сети позволяют увеличить точность прогноза в два и более раза по сравнению с точностью прогноза, даваемой традиционным методом экстраполяции по скользящей средней.
\end{abstract}

\section{Ключевые слова:}

Месторождение нефти и газа, добывающая скважина, прогноз значений дебитов скважин,

модели глубоких искусственных нейронных сетей,

исследование эффрективности глубоких нейронных сетей прямого распространения.

\section{Введение}

При добыче углеводородного сырья (УВС) на промыслах происходит измерение большого числа технологических и геологических параметров. Значения этих параметров служат для оценки состояния скважин и продуктивных пластов, а также позволяют понять, какова технологическая и экономическая эффективность проведённых работ на фонде скважин, в том числе и геолого-технических мероприятий (ГТМ).

Задача прогноза значений параметров актуальна не только для расчёта технологических режимов скважин, но и для управления фондом скважин, так как выявленный критический тренд может позволить заранее установить необходимость проведения определённого вида ГТМ на скважине [1]. Однако существующие методы прогноза значений дебитов скважины в лучшем случае позволяют выполнить экстраполяцию данных путём линейной регрессии [2, 3]. Разумеется, столь простые методы не способны учесть множество аспектов, заложенных в данных, и взаимовлияние их друг на друга в рамках получаемых временных рядов для параметров скважин [4]. Соот- ветственно, точность прогноза не всегда удовлетворяет специалистов нефтегазодобывающих предприятий. Сделаны первые шаги в использовании методов интеллектуального анализа данных при решении таких задач, давшие первые обнадёживающие результаты [5-7].

Всё это указывает на актуальность разработки новых методов интеллектуального анализа данных, позволяющих существенного повысить точность прогноза значений параметров скважин и продуктивных пластов.

В данной работе для прогноза значений дебитов скважин было предложено использовать глубокие искусственные нейронные сети (ИНС) [8], показавшие свою эффективность при решении задач экстраполяции данных в других предметных областях. Проведено исследование эффективности различных архитектур глубоких ИНС прямого распространения при решении таких задач прогноза. Результаты исследований показали, что использование этих ИНС позволяет значительно повысить точность прогноза значений дебитов скважин по сравнению с точностью, достигаемой с помощью известных методов. 


\section{Постановка задачи исследования}

Целью планирования работы производства нефтегазодобывающего предприятия является определение таких технологических режимов работы фонда скважин и технологического оборудования, которые позволят достичь заданного объёма выпуска продукции (нефти, газа и газового конденсата). При этом наиболее важно планирование технологических режимов каждой добывающей скважины фонда и технологических режимов установок подготовки продукции из добываемого УВС, а также планирование режимов транспортировки продукции до магистральных нефте- и газопроводов.

Обычно на эксплуатируемых месторождениях прогнозу подлежит довольно большое число измеряемых параметров добывающих скважин и продуктивных пластов, среди них: дебит нефти, дебит газа, дебит жидкости (конденсата), дебит воды, давление забойное, давление пластовое, обводнённость и т. д. Однако наиболее востребованными являются прогнозы значений дебитов скважин.

Точное предсказание дебитов скважин является необходимой частью управления производством любого нефтегазодобывающего предприятия. Важно прогнозировать (планировать) дебиты не просто на конкретный день, а на определённый промежуток времени (например, равный месяцу), чтобы понимать, сколько в среднем УВС будет добываться из скважины в сутки. В этом случае говорят о планировании технологического режима скважины. Значение технологического режима скважины позволяет рассчитать экономический эффект от добычи УВС и понять рентабельность эксплуатации конкретной скважины, построить перспективные планы поставок продукции и учесть ограничения, наложенные органами надзора за пользование недрами [9].

Для обеспечения высокой точности прогноза значений дебитов скважины было предложено использовать глубокие ИНС прямого распространения. Поэтому была поставлена задача исследования эффективности таких ИНС при решении задачи прогноза значений дебитов при использовании реальных данных с эксплуатируемого месторождения.

Для проведения исследований использовался архив данных одного из нефтегазоконденсатных месторождений Томской области, где за 6 лет эксплуатации накоплен значительный объём данных. Фонд добывающих скважин содержит 17 кустов скважин, в каждом кусте от 6 до 21 добывающей скважины, всего 142 добывающих скважины.

Для экспериментов были выбраны исключительно параметры типа дебитов (ежесуточные объёмы добычи компонентов УВС - нефти, воды и т. д.), так как они являются наиболее значимыми интегральными показателями добычи УВС. Для них в базе данных (БД) предприятия обычно имеется наибольший объём данных, не является исключением и предприятие, из архива которого были взяты данные для исследований.

\section{Методика подготовки данных для обучения и тестирования искусственных нейронных сетей}

На предприятии использовалась методика прогноза (планирования) значений дебитов, основанная на методе экстраполяции по скользящей средней $[10,11]$. Для каждого из дебитов скважин в анализируемой БД имеются прогнозное и фактическое значения. Прогноз геологической службой предприятия, судя по имеющимся данным, осуществлялся от -8 до 229 дней до измерения фактического значения дебита. То есть в БД имеются явно ошибочные значения параметров, по крайней мере, при планировании значений дебитов, так как нельзя планировать значение параметра уже после получения его фактического значения. Также вызывают сомнения прогнозы на большие временные промежутки (на 2 и более месяцев). Все такие данные отбраковывались и в экспериментах не использовались [12].

Bce фактические измеренные значения дебитов на дату планирования и ранее подаются на входы ИНС (признак, англ. feature). Фактические значения дебитов через месяц после этой даты являются эталоном, то есть они служат эталоном выходным значениям ИНС (метка, англ. label) при её обучении и тестировании.

Данные, которые находятся между датой планирования и более поздней датой получения фактического значения дебита-эталона, не используются, несмотря на их наличие в БД, чтобы находиться в тех же самых условиях, что и находилась геологическая служба предприятия, планировавшая технологический режим работы скважины. Это позволит корректно осуществить сравнение полученных при исследовании результатов с имеющимися в БД значениями прогнозных (плановых) дебитов.

При проведении экспериментов было решено брать значения дебитов от даты планирования в течение трёх-пяти недель до получения фактического значения. Это практически важно при формировании технологических режимов скважин. Исходное число записей в БД 17117, после сужения периода планирования до заданного записей осталось 13032. Видно, что такие действия уменьшили объём обучающей выборки. Однако это позволило нам получить более точную оценку результатов прогноза значений дебитов на месяц по существующей на предприятии методике.

Число анализируемых записей и рассчитанные нами значения взвешенных средних абсолютных ошибок (англ. Weighted absolute percent error - WAPE) [13] при прогнозе каждого параметра (вида дебита) по всему месторождению по методике предприятия указаны в табл. 1. При разработке наших моделей ИНС и при оценке их точности необходимо ориентироваться на уменьшение WAPE при прогнозе значений этих дебитов.

Текущий или капитальный ремонт скважин, остановка их по другим причинам или на проведение ГТМ вносят непредсказуемость в изменение параметров скважин. Это должно быть учтено при составлении обучающих примеров, чтобы в вектор признаков на входах ИНС по каждой скважине не попадали зна- 
чения дебитов одновременно до и после проведения тех или иных ГТМ или остановок скважин. То есть планирование технологического режима скважины должно осуществляться исключительно с учетом периодов её эксплуатации.

Таблица 1. Оиенки точности прогноза параметров по методике предприятия

Table 1. Estimates of the forecasting precision of parameters by the enterprise method

\begin{tabular}{|c|c|c|c|c|}
\hline $\begin{array}{c}\text { Название пара- } \\
\text { метра } \\
\text { Name of } \\
\text { parameter }\end{array}$ & 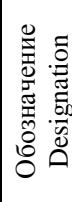 & $\begin{array}{c}\text { Единица } \\
\text { измерения } \\
\text { Measuring } \\
\text { unit }\end{array}$ & 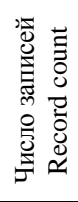 & $\begin{array}{c}\text { Взвешенная } \\
\text { средняя абсо- } \\
\text { лютная } \\
\text { ошибка, \% } \\
\text { Weighted absolute } \\
\text { percent error, \% }\end{array}$ \\
\hline $\begin{array}{l}\text { дебит нефти } \\
\text { debit of oil }\end{array}$ & $\begin{array}{l}Q_{\text {н }} \\
Q_{\text {。 }}\end{array}$ & $\begin{array}{c}\mathrm{T} / \mathrm{cyT} \\
\text { tons/day }\end{array}$ & 3557 & 27,347 \\
\hline $\begin{array}{l}\text { дебит газа } \\
\text { debit of gas }\end{array}$ & $\begin{array}{l}Q_{\text {г }} \\
Q_{\mathrm{g}}\end{array}$ & $\begin{array}{l}\text { тыс. } \mathrm{m}^{3} / \text { сут } \\
1000 \mathrm{~m}^{3} / \text { day }\end{array}$ & 3458 & 35,133 \\
\hline $\begin{array}{l}\text { дебит жидкости } \\
\text { debit of fluid }\end{array}$ & $\begin{array}{l}Q_{\text {ж }} \\
Q_{\mathrm{f}}\end{array}$ & $\begin{array}{l}\mathrm{m}^{3} / \text { cyт } \\
\mathrm{m}^{3} / \text { day }\end{array}$ & 3459 & 26,734 \\
\hline $\begin{array}{l}\text { дебит воды } \\
\text { debit of water }\end{array}$ & $\begin{array}{l}Q_{\mathrm{B}} \\
Q_{\mathrm{w}}\end{array}$ & $\begin{array}{c}\mathrm{T} / \mathrm{cyT} \\
\text { tons/day }\end{array}$ & 2558 & 67,044 \\
\hline
\end{tabular}

Не ясно, какое оптимальное число значений дебита нужно подавать на входы ИНС, это предстоит определить экспериментально. Также открытым остаётся вопрос, стоит ли подавать на входы ИНС значения только одного какого-либо дебита или добавлять к ним ещё значения других параметров, например, дебита другой компоненты УВС или пластового давления продуктивного пласта. Так как число таких сочетаний очень велико, подобные эксперименты не выполнялись, и в качестве входных значений для ИНС использовались только фактические значения прогнозируемого дебита.

Суть предлагаемой методики подготовки данных в том, чтобы увеличивать при прогнозе число входных значений дебитов для ИНС за счёт расширения числа известных из БД фактических значений дебитов от 6 (6 дней назад от даты планирования) с постепенным добавлением таких значений за предыдущие дни до 15. Схематично процесс выбора данных изображён на рис. 1, где показано движение скважины во времени. Видно, что скважина была два раза остановлена на разные промежутки времени. Жирными красными штрихами отмечены взятые в эксперимент 13 фактических значений дебита на скважине, получаемые ежедневно. Пусть дата прогноза значения дебита будет отмечена знаком «!» (метка для ИНС), и на эту дату в БД должно быть фактическое значение дебита. Дата, с которой планируется значение дебита, показана знаком «?». С даты планирования и по дату прогноза должен пройти один месяц (период планирования). Все фактические значения дебита (от 6 до 15 значений) до даты планирования становятся признаками, подаваемыми на входы ИНС (отмечены жирными красными штрихами).

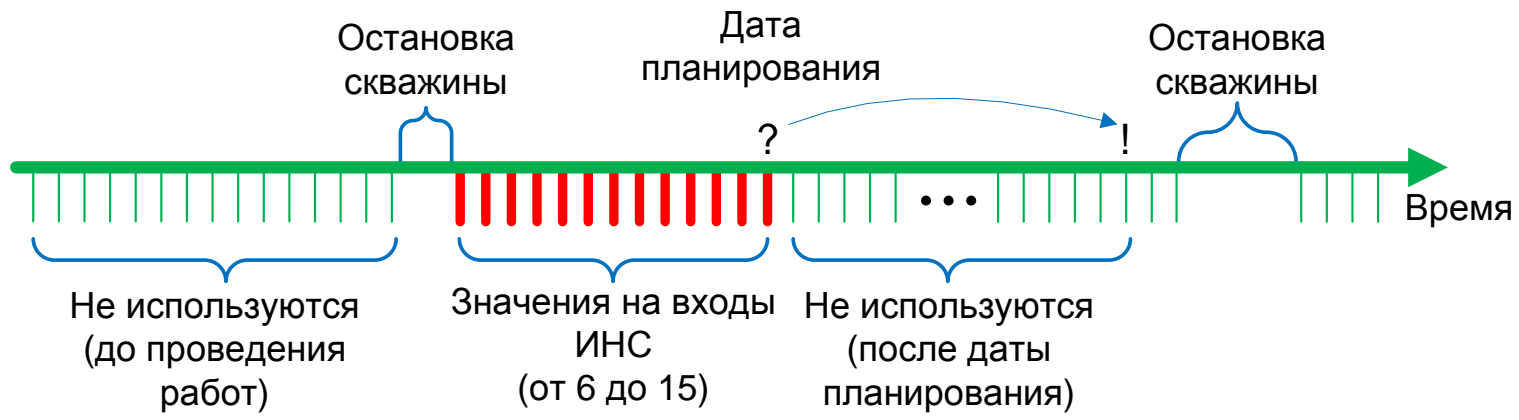

Pис. 1. Схема выбора значений измеряемых дебитов

Fig. 1. Selection scheme of values for the measured debits

Дате планирования может предшествовать остановка скважины для проведения каких-либо работ (например, ГТМ). Поэтому число признаков для ИНС, большее некоторого имеющегося числа, взять не получится. Например, из рис. 1 следует, что выбрать 14 признаков не удастся. Это значит, что в общем случае обучающие выборки будут несопоставимы по объёму, так как для малого числа признаков на входах ИНС (например, 6) будет всегда больше обучающих примеров, чем для большого числа признаков (например, 15).

Если за какой-либо день в БД отсутствует фактическое значение дебита, и это не связано с проведением работ на скважине, то производится линейная интерполяция значения дебита по соседним известным значениям. Использование линейной интерполяции было продиктовано разработчиками БД.

Если между двумя остановками скважины есть только одно измерение дебита, или оно вообще отсутствует, то в таких условиях провести интерполяцию нельзя, и прогноз на этом интервале становится невозможным [14]. Если на скважине ГТМ не проводились, то берётся весь массив значений фактических дебитов для анализа без каких-либо ограничений. Аналогичным образом для анализа используются все значения параметров после последнего проведённого ГТМ на скважине.

Для извлечения данных о дебитах и остановках скважин, в том числе для ГТМ, из БД было создано специальное программное обеспечение (ПО). Данное ПО позволяет провести всю необходимую предварительную обработку данных, выбрать вид дебита (дебит нефти, газа, жидкости (газового конденсата) или воды), число признаков на входе ИНС (от 6 до 15), 
указать число блоков для кросс-валидации данных методом К блоков [15], а также создать файл для последующего обучения или тестирования ИНС. Пользователь видит при загрузке данных информацию о характеристиках выборки в текстовом поле. При формировании файла указывается, сколько было задействовано скважин при построении выборки и общее число обучающих и тестовых примеров.

Для проведения экспериментов с помощью этого ПО было построено 40 пар файлов (для обучения и тестирования ИНС) - по 10 пар на каждый вид дебита. Объёмы пар «признаки-метка» для разного числа входов ИНС и для всех дебитов указаны в табл. 2 . При использовании метода К блоков выбрано К=5. Это означает, что все вычисленные в ходе исследований итоговые значения WAPE прогноза дебитов являются результатом усреднения по пяти значениям ошибок, полученным в ходе обучения и тестирования ИНС.

Таблица 2. Объёмы выборок для различных дебитов и разного числа входов искусственной нейронной сети

Table 2. Volumes of selections for various debits and different number of artificial neural network inputs

\begin{tabular}{|c|c|c|c|c|c|c|c|c|c|c|}
\hline \multirow{2}{*}{$\begin{array}{l}\text { Дебиты } \\
\text { Debits }\end{array}$} & \multicolumn{10}{|c|}{$\begin{array}{c}\text { Число входов ИНС } \\
\text { Number of ANN inputs }\end{array}$} \\
\hline & 6 & 7 & 8 & 9 & \begin{tabular}{|l|}
10 \\
\end{tabular} & \begin{tabular}{|l|}
11 \\
\end{tabular} & \begin{tabular}{|l|}
12 \\
\end{tabular} & 13 & 14 & 15 \\
\hline $\begin{array}{l}Q_{\mathrm{H}} \\
Q_{\mathrm{o}}\end{array}$ & 1248 & 1248 & 1246 & 1246 & 1244 & 1244 & 1243 & 1243 & 1243 & 1235 \\
\hline $\begin{array}{l}Q_{\mathrm{r}} \\
Q_{\mathrm{g}}\end{array}$ & 1199 & 1199 & 1197 & 1197 & 1195 & 1195 & 1194 & 1194 & 1194 & 1188 \\
\hline $\begin{array}{l}Q_{\text {ж }} \\
Q_{\mathrm{f}} \\
\end{array}$ & 1199 & 1199 & 1197 & 1197 & 1195 & 1195 & 1194 & 1194 & 1194 & 1188 \\
\hline $\begin{array}{l}Q_{\mathrm{B}} \\
Q_{\mathrm{w}}\end{array}$ & 880 & 877 & 875 & 875 & 873 & 873 & 872 & 872 & 872 & 868 \\
\hline
\end{tabular}

\section{Эксперименты по прогнозу значений дебитов скважин}

Программная реализация ИНС для проведения экспериментов выполнена на языке С\# с использованием библиотеки Microsoft CNTK [16], имеющей определённые преимущества перед другими библиотеками $[17,18]$. С её помощью были автоматизированы операции по изменению архитектуры и гиперпараметров ИНС, обучены и тестированы ИНС на вышеуказанных выборках.

Число скрытых слоёв ИНС прямого распространения выбиралось равным 2, 3, 4, 5, 6, 7, 8, 10, 15. В ходе экспериментов было выявлено, что число слоёв больше восьми не даёт прироста точности прогноза. Исследовалось также применение различных функций активации (TanH, LeakyReLU) [19], позволяющих получать связи между нейронами с отрицательными воздействиями. Алгоритм оптимизации при обучении ИНС Adam [20]. Скорость обучения динамически определялась по этому алгоритму. Каждый обучающий пример уникален и должен осуществлять воздействия индивидуально, но скорость обучения тем больше, чем меньше объём минивыборки, поэтому был выбран компромиссный вариант, равный 5. Число эпох выбиралось динамически, в соответствии с достигаемой точностью обучения, если она несколько эпох существенно не менялась. Число нейронов в каждом скрытом слое выбиралось в соответствии с эвристическим правилом: Число Нейронов = Число Входов * $2+2$.

На входы ИНС подаются фактические значения дебита за определённое число дней (от 6 до 15). Далее идёт ряд скрытых слоёв ИНС - от 2 до 8. В выходном слое ИНС один нейрон (прогнозируемое значение дебита). На рис. 2 в качестве примера приведена архитектура ИНС с 3 скрытыми слоями, 12 фактическими значениями дебита на входах ИНС и 26 нейронами в скрытых слоях, функция активации LeakyReLU.

\section{Фактические значения дебита за 12 дней

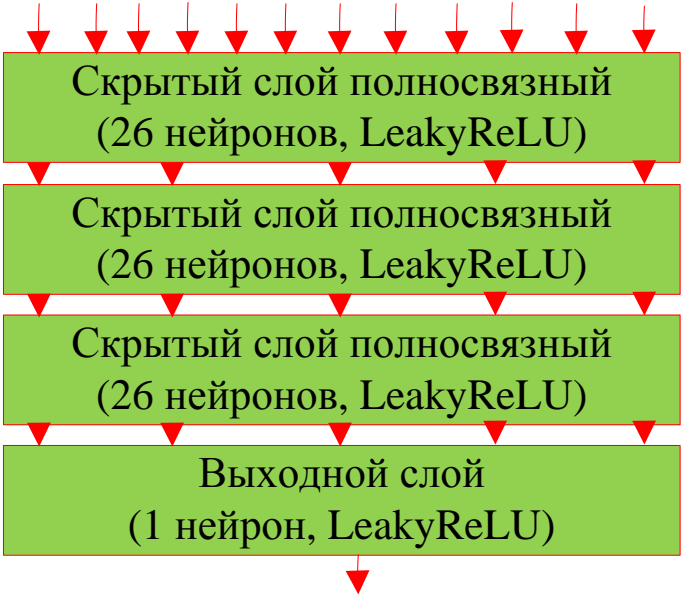 \\ Прогнозное значение дебита}

Pис. 2. Схема архитектуры искусственной нейронной сети Fig. 2. Architecture scheme of an artificial neural network

В табл. 3 приведены в качестве примера результаты экспериментов с ИНС различных архитектур при решении на тестовых выборках задачи прогноза значений дебита нефти $Q_{\mathrm{H}}$. При этом переменными являются как функции активации и число скрытых слоёв ИНС, так и число входов ИНС. Значение ошибки прогноза вычисляется как среднее по всем ошибкам, полученным по схеме кросс-валидации по 5 блокам, и по всем ошибкам для добывающих скважин в выборках. Жирным отмечается лучшая точность, результаты округлены до тысячных. Аналогичные эксперименты на тестовых выборках были проведены для дебитов газа, жидкости и воды, их результаты сведены в три таблицы.

Такие же четыре таблицы содержат результаты прогноза значений дебитов в случае обучающих выборок. Отметим, что значения WAPE на обучающих выборках меньше до 30 \% по сравнению с ошибками, полученными с помощью ИНС на тестовых выборках. Причём чем больше число входов и число слоёв ИНС, тем меньше разница WAPE, полученных на обучающей и тестовой выборках.

Лучшие результаты из всех четырёх таблиц со значениями WAPE при прогнозе дебитов, полученные при исследованиях ИНС с различными архитектурами на тестовых выборках, занесены в табл. 4. 
Сравнительный анализ лучших результатов из табл. 4 c WAPE прогноза из табл. 1 показал, что для каждого из дебитов точность прогноза с помощью ИНС выше, чем полученная с помощью метода экстраполяции по скользящей средней. Прирост точности прогноза дебитов с помощью ИНС указан для тестовых выборок в сравнении с результатами из табл. 1 в последнем столбце табл. 4. Видно, что лучший результат прогноза каждого из дебитов, полученный с использованием ИНС, в два и более раза точнее, чем результат прогноза каждого из дебитов по методике, принятой на предприятии.

Таблица 3. Результаты прогноза $Q_{н}$ с помощью искусственной нейронной сети на тестовых выборках

Table 3. Results of forecasting $Q_{o}$ by means of artificial neural network on test selections

\begin{tabular}{|c|c|c|c|c|c|c|c|c|c|}
\hline \multirow{2}{*}{$\begin{array}{l}\text { Параметр } \\
\text { Parameter }\end{array}$} & \multirow{2}{*}{$\begin{array}{l}\text { Число входов ИНС } \\
\text { Number of ANN inputs }\end{array}$} & $\begin{array}{l}\text { Функция активации } \\
\text { Activation function }\end{array}$ & \multicolumn{7}{|c|}{$\begin{array}{l}\text { Взвешенная средняя абсолютная ошибка, \% } \\
\text { Weighted absolute percent error, \% }\end{array}$} \\
\hline & & \begin{tabular}{|c|} 
Число слоёв \\
Number of layers \\
\end{tabular} & 2 & 3 & 4 & 5 & 6 & 7 & 8 \\
\hline \multirow{20}{*}{$\begin{array}{l}Q_{\text {н }} \\
Q_{\text {о }}\end{array}$} & \multirow{2}{*}{6} & TanH & 16,705 & 16,841 & 17,775 & 15,163 & 16,695 & 17,893 & 16,705 \\
\hline & & LeakyReLU & 20,538 & 17,978 & 18,385 & 16,645 & 18,146 & 17,445 & 20,538 \\
\hline & \multirow{2}{*}{7} & TanH & 18,197 & 17,723 & 17,356 & 17,617 & 15,420 & 17,109 & 18,197 \\
\hline & & LeakyReLU & 18,545 & 17,942 & 17,563 & 17,500 & 17,123 & 15,777 & 18,545 \\
\hline & \multirow{2}{*}{8} & TanH & 16,282 & 17,384 & 17,141 & 17,147 & 20,219 & 19,081 & 16,282 \\
\hline & & LeakyReLU & 16,993 & 17,496 & 17,621 & 16,837 & 16,168 & 17,370 & 16,993 \\
\hline & \multirow{2}{*}{9} & TanH & 16,551 & 17,380 & 17,356 & 18,837 & 17,678 & 15,810 & 16,551 \\
\hline & & LeakyReLU & 17,040 & 18,290 & 16,720 & 18,991 & 15,858 & 15,467 & 17,040 \\
\hline & \multirow{2}{*}{10} & TanH & 14,127 & 14,674 & 12,623 & 13,296 & 13,985 & 14,523 & 13,298 \\
\hline & & LeakyReLU & 13,889 & 13,849 & 12,477 & 13,337 & 13,456 & 13,351 & 13,768 \\
\hline & \multirow{2}{*}{11} & TanH & 13,236 & 12,794 & 14,579 & 14,174 & 13,643 & 16,728 & 13,236 \\
\hline & & LeakyReLU & 13,718 & 13,463 & 13,680 & 12,685 & 13,222 & 13,438 & 13,718 \\
\hline & \multirow{2}{*}{12} & TanH & 15,568 & 16,006 & 15,149 & 17,024 & 16,967 & 16,413 & 15,568 \\
\hline & & LeakyReLU & 15,858 & 15,660 & 15,712 & 15,617 & 16,456 & 15,315 & 15,858 \\
\hline & \multirow{2}{*}{13} & TanH & 16,869 & 15,179 & 15,378 & 16,124 & 16,000 & 16,430 & 16,869 \\
\hline & & LeakyReLU & 16,541 & 15,398 & 14,602 & 15,631 & 14,332 & 15,240 & 16,541 \\
\hline & \multirow{2}{*}{14} & TanH & 15,637 & 15,179 & 15,962 & 15,968 & 16,270 & 16,099 & 15,637 \\
\hline & & LeakyReLU & 17,494 & 15,988 & 14,995 & 15,611 & 14,843 & 15,019 & 17,494 \\
\hline & \multirow{2}{*}{15} & TanH & 15,360 & 15,224 & 14,962 & 17,200 & 15,052 & 15,123 & 15,360 \\
\hline & & LeakyReLU & 15,372 & 14,731 & 14,427 & 14,756 & 14,050 & 13,868 & 15,372 \\
\hline
\end{tabular}

Таблица 4. Лучшие результаты при прогнозе значений дебитов скважин с помощью искусственной нейронной сети

Table 4. The best results at forecasting of wells debit values by means of artificial neural network

\begin{tabular}{|c|c|c|c|}
\hline $\begin{array}{l}\text { Параметр } \\
\text { Parameter }\end{array}$ & $\begin{array}{c}\text { Взвешенная средняя абсолютная } \\
\text { ошибка, \% } \\
\text { Weighted absolute percent error, \% }\end{array}$ & $\begin{array}{l}\text { Особенности архитектуры ИНС } \\
\text { Features of ANN architecture }\end{array}$ & $\begin{array}{c}\text { Прирост точности прогноза, \% } \\
\text { Boost of the forecasting } \\
\text { precision, } \%\end{array}$ \\
\hline $\begin{array}{l}Q_{\text {н }} \\
Q_{\mathrm{o}}\end{array}$ & 12,477 & $\begin{array}{c}10 \text { входов ИНС; } 4 \text { скрытых слоя; функция } \\
\text { активации LeakyReLU } \\
10 \text { inputs of ANN; } 4 \text { hidden layers; activation } \\
\text { function LeakyReLU }\end{array}$ & 54,438 \\
\hline $\begin{array}{l}Q_{\text {г }} \\
Q_{\mathrm{g}}\end{array}$ & 17,129 & $\begin{array}{c}11 \text { входов ИНС; } 6 \text { скрытых слоёв; функция } \\
\text { активации LeakyReLU } \\
11 \text { inputs of ANN; } 6 \text { hidden layers; activation } \\
\text { function LeakyReLU }\end{array}$ & 51,245 \\
\hline $\begin{array}{l}Q_{\text {ж }} \\
Q_{\mathrm{f}}\end{array}$ & 13,584 & $\begin{array}{c}10 \text { входов ИНС; } 7 \text { скрытых слоёв; функция } \\
\text { активации LeakyReLU } \\
10 \text { inputs of ANN; } 7 \text { hidden layers; activation } \\
\text { function LeakyReLU }\end{array}$ & 49,188 \\
\hline $\begin{array}{l}Q_{\text {в }} \\
Q_{\mathrm{w}}\end{array}$ & 29,513 & $\begin{array}{c}10 \text { входов ИНС; } 7 \text { скрытых слоёв; функция } \\
\text { активации LeakyReLU } \\
10 \text { inputs of ANN; } 7 \text { hidden layers; activation } \\
\text { function LeakyReLU }\end{array}$ & 55,980 \\
\hline
\end{tabular}

\section{Заключение}

При планировании показателей производства нефтегазодобывающего предприятия важным является прогноз значений дебитов каждой добывающей скважины. Для повышения точности такого прогноза было предложено использовать глубокие искусственные нейронные сети.

Разработана методика подготовки реальных данных по дебитам скважин для обучения и тестирования глубоких искусственных нейронных сетей прямо- го распространения. Проведены исследования различных архитектур таких искусственных нейронных сетей при решении задач прогноза дебитов нефти, газа, жидкости (газового конденсата) и дебита воды. В результате этих исследований выявлены наиболее эффективные архитектуры искусственных нейронных сетей, позволяющие увеличить точность прогноза в два и более раза по сравнению с точностью прогноза, даваемой традиционным методом экстраполяции по скользящей средней. 


\section{СПИСОК ЛИТЕРАТУРЫ}

1. Анкудинов А.А., Полякова Н.С., Радевич Ю.E. LUKOIL: Moниторинг разработки месторождения с использованием статистических методов анализа на примере перфорационных работ // ROGTEC Field Development, 2019. URL: https://rogtecmagazine.com/wpcontent/uploads/2019/12/LUKOIL-How-Data-Mining-EnhancesOilfield-Development.pdf (дата обращения 26.06.2020).

2. Марков Н.Г. Информационно-управляющие системы для газодобывающего производства. - Томск: Изд-во Томского политехнического университета, 2016. - $261 \mathrm{c}$.

3. Improvement of technological-mathematical model for the medium-term prediction of the work of a gas condensate field / $\mathrm{M}$ Kutia, M. Fyk, O. Kravchenko, S. Palis, I. Fyk // Eastern-European Journal of Enterprise Technologies. - 2016. - V. 83. - P. 40-48.

4. Косков В.Н. Геофизические исследования скважин. - Пермь: Пермский государственный технический университет, 2004. $122 \mathrm{c}$.

5. Евсюткин И.В., Марков Н.Г. Управление геологотехническими мероприятиями на месторождениях нефти и газа с использованием искусственных нейронных сетей // Доклады ТУСУР. - 2020. - Т. 23. - № 1. - С. 62-69.

6. Al-Fattah S.M., Startzman R. Predicting natural gas production using artificial neural network // 2001 SPE Hydrocarbon Economics and Evaluation Symposium, SPE 68593. Dallas, TX, USA. URL: https://www.researchgate.net/publication/ 261697475_Predicting_Natural_Gas_Production_Using_Artificial Neural Network (дата обращения 26.06.2020).

7. Новый подход к доуточнению прогнозов прокси-моделей пласта с помощью алгоритмов машинного обучения / О.В. Зоткин, М.В. Симонов, А.Е. Осокина, А.М. Андрианова, А.С. Маргарит, В.А. Тимошенко, С.Ф. Лебедев // Нефтяное хозяйство. - 2019. - № 12. - С. 60-63.

8. Khandakar M.R., Louis J. Times-series data augmentation and deep learning for construction equipment activity recognition // Advanced Engineering Informatics. - 2019. - V. 42. - P. 1-12.

9. Раджабов С.Р., Базаров Г.Р. Технологический режим эксплуатации скважин месторождения Зеварды // Молодой ученый. 2015. - T. 88. - № 8. - C. 292-294. URL: https://moluch.ru/ archive/88/16556/ (дата обращения: 26.06.2020).
10. Generating moving average trading rules on the oil futures market with genetic algorithms / L. Wang, H. An, X. Xia, X. Liu, X. Sun, X. Huang // Mathematical Modelling and Algorithms in Finance. 2014. - V. 2014. - 10 p. [Article ID 101808].

11. Вальес-Лосано Дж., Репин-Поляков Н.Н. Применение метода кривой падения Арпса для прогноза дебита скважины // Наука, техника и образование. - 2020. - T. 69. - № 5. URL: https://cyberleninka.ru/article/n/primenenie-metoda-krivoypadeniya-arpsa-dlya-prognoza-debita-skvazhiny (дата обращения: 26.06.2020).

12. Kofi Sarpong Adu-Manu, John Kingsley Arthur. A review of data cleansing concepts achievable goals and limitations // International Journal of Computer Applications. - 2013. - V. 7. - № 76. P. 19-22.

13. A survey of forecast error measures / M. Shcherbakov, N.L. Shcherbakova, A. Brebels, A. Tyukov // World Applied Sciences Journal. - 2013. - V. 24. - P. 171-176.

14. Bourke P. Interpolation methods. 1999. URL: http://paulbourke. net/miscellaneous/interpolation/ (дата обращения: 26.06.2020).

15. Berrar D. Cross-Validation // Encyclopedia of Bioinformatics and Computational Biology. - 2018. - V. 1. - P. 542-545.

16. The Microsoft Cognitive Toolkit. URL: https://docs.microsoft. com/en-us/cognitive-toolkit/ (дата обращения 26.06.2020).

17. Benchmarking state-of-the-art deep learning software tools / Shaohuai Shi, Qiang Wang, Pengfei Xu, Xiaowen Chu // arXiv:1608.07249v7 [cs. DC] 17 Feb 2017. - URL: https://arxiv.org/pdf/1608.07249.pdf (дата обращения: 26.06.2020).

18. Григоренко Е. «Почему CNTK?». URL: https://habr.com/ru/ company/microsoft/blog/336552/ (дата обращения: 26.06.2020).

19. «7 Types of Neural Network Activation Functions: How to Choose?». URL: https://missinglink.ai/guides/neural-networkconcepts/7-types-neural-network-activation-functions-right/ (дата обращения: 26.06.2020).

20. Baptista D., Rodrigues S., Morgado-Dias F. Performance comparison of ANN training algorithms for classification // Conference: Intelligent Signal Processing (WISP). IEEE 8th International Symposium. - Funchal, Portugal, 2013. - P. 115-120.

Поступила 03.11.2020 2.

\section{Информация об авторах}

Евсюткин И.В., ассистент отделения информационных технологий Инженерной школы информационных технологий и робототехники Национального исследовательского Томского политехнического университета.

Марков Н.Г., доктор технических наук, профессор отделения информационных технологий Инженерной школы информационных технологий и робототехники Национального исследовательского Томского политехнического университета. 
UDC 622.276:004.896

\title{
DEEP ARTIFICIAL NEURAL NETWORKS FOR FORECASTING DEBIT VALUES FOR PRODUCTION WELLS
}

\author{
Ivan V. Evsyutkin', \\ ive1@tpu.ru
}

\author{
Nikolay G. Markov', \\ markovng@tpu.ru \\ 1 National Research Tomsk Polytechnic University,
30, Lenin avenue, Tomsk, 634050, Russia.
}

The relevance of the research is caused by the need for supporting the decision-making by specialists of the extracting enterprises of the oil and gas industry at production management. Precise forecasting of production wells debits values allows defining such technological operating modes of a well stock and processing equipment which would allow reaching the prescribed production volume. The existing methods do not always provide the demanded precision level at the forecast of wells debits values that leads to mistakes at the calculation of economic effect at a profitability assessment of production wells and the subsequent deliveries of hydrocarbonic raw materials, and also at taking into account the restrictions imposed by environmental supervision of natural resources use.

The main aim of the research is to develop and offer the most effective models of deep artificial neural networks at the forecast of production components values for hydrocarbonic raw materials - oil, gas, fluid (gas condensate), and water.

Objects of the research are technological parameters of production wells debits of well-stock of oil, gas, oil and gas, and oil-gas condensate fields.

Methods: the methods of the analysis of a large amount of technical data of wells developed according to the concept of «Big Data»; models of deep artificial neural networks; object-oriented programming; methods of an assessment and the statistical analysis of research results of deep artificial neural networks efficiency at the forecast of production wells debit values.

Results. The technique of data preparation for wells debits is developed for training and testing of feed-forward deep artificial neural networks. Research is carried out for various architectures for such artificial neural networks at the solution of the forecasting task of oil, gas, fluid (gas condensate), and water debits. The most effective architecture of feed-forward deep artificial neural networks is developed. Such neural networks allow increasing the forecasting accuracy in two and more times in comparison with the accuracy of the forecasting received by a traditional method of extrapolation (moving average).

\section{Key words:}

Oil and gas field, production well, forecasting of wells debits values, models of deep artificial neural networks, research of efficiency of feed-forward deep neural networks.

\section{REFERENCES}

1. Ankudinov A.A., Polyakova N.S., Radevich Y.E. LUKOIL: How Data Mining Enhances Oilfield Development. ROGTEC Field Development, 2019. Available at: https://rogtecmagazine.com/wpcontent/uploads/2019/12/LUKOIL-How-Data-Mining-EnhancesOilfield-Development.pdf (assessed 26 June 2020).

2. Markov N.G. Informatsionno-upravlyayushchie sistemy dlya gazodobyvayushchego proizvodstva [Management information systems for gas production]. Tomsk, Tomsk Polytechnic University Publ. house, 2016. $261 \mathrm{p}$.

3. Kutia M., Fyk M., Kravchenko O., Palis S., Fyk I. Improvement of technological-mathematical model for the medium-term prediction of the work of a gas condensate field. Eastern-European Journal of Enterprise Technologies, 2016, vol. 83, pp. 40-48.

4. Koskov V.N. Geofizicheskie issledovaniya skvazhin [Geophysical surveys of wells]. Perm, Perm State Technical University Publ., 2004. $122 \mathrm{p}$.

5. Evsyutkin I.V., Markov N.G. Upravlenie geologo-tekhnicheskimi meropriyatiyami na mestorozhdeniyakh nefti i gaza s ispolzovaniem iskusstvennykh neyronnykh setey [Management of geological and technical arrangements at oil and gas fields with the use of artificial neural networks]. Doklady TUSUR, 2020, vol. 23, no. 1 , pp. 62-69.

6. Al-Fattah S.M., Startzman R. Predicting natural gas production using artificial neural network. 2001 SPE Hydrocarbon Economics and Evaluation Symposium, SPE 68593. Dallas, TX, USA. Available at: https://www.researchgate.net/publication/ 261697475_Predicting_Natural_Gas_Production_Using_Artificial _Neural_Network (assessed 26 June 2020).

7. Zotkin O.V., Simonov M.V., Osokina A.E., Andrianova A.M., Margarit A.S., Timoshenko V.A., Lebedev S.F. Novy podkhod k doutochneniyu prognozov proksi-modeley plasta s pomoshchyu algoritmov mashinnogo obucheniya [New approach to the additional improvement of forecasts of layer proxy models using machine learning algorithms]. Neftyanoe hozyaystvo, 2019, no. 12, pp. 60-63.

8. Khandakar M.R., Louis J. Times-series data augmentation and deep learning for construction equipment activity recognition. Advanced Engineering Informatics, 2019, vol. 42, pp. 1-12.

9. Radzhabov S.R., Bazarov G.R. Tekhnologicheskiy rezhim ekspluatatsii skvazhin mestorozhdeniya Zevardy [Technological mode of the wells' operation of Zevarda field]. Molodoy Ucheniy, 2015, vol. 88, no. 8, pp. 292-294. Available at: https://moluch.ru/archive/88/16556/ (assessed 26 June 2020).

10. Wang L., An H., Xia X., Liu X., Sun X., Huang X. Generating moving average trading rules on the oil futures market with genetic algorithms. Mathematical Modelling and Algorithms in Finance, 2014, vol. 2014, 10 p. [Article ID 101808].

11. Valyes-Losano J., Repin-Polyakov N.N. Primenenie metoda krivoy padeniya Arpsa dlya prognoza debita skvazhiny [Application of the Arps decline curve method for the forecasting of well debit]. Nauka, tekhnika i obrazovanie, 2020, vol. 69, no. 5. Available at: https://cyberleninka.ru/article/n/primenenie-metodakrivoy-padeniya-arpsa-dlya-prognoza-debita-skvazhiny (assessed 26 June 2020).

12. Kofi Sarpong Adu-Manu, John Kingsley Arthur. A review of data cleansing concepts achievable goals and limitations. International Journal of Computer Applications, 2013, vol. 7, no. 76, pp. 19-22.

13. Shcherbakov M., Shcherbakova N.L., Brebels A., Tyukov A. A survey of forecast error measures. World Applied Sciences Journal, 2013, vol. 24, pp. 171-176.

14. Bourke P. Interpolation methods. 1999. Available at: http:// paulbourke.net/miscellaneous/interpolation/ (assessed 26 June 2020). 
15. Berrar D. Cross-Validation. Encyclopedia of Bioinformatics and Computational Biology, 2018, vol. 1, pp. 542-545.

16. The Microsoft Cognitive Toolkit. Available at: https://docs. microsoft.com/en-us/cognitive-toolkit/ (assessed 26 June 2020).

17. Shaohuai Shi, Qiang Wang, Pengfei Xu, Xiaowen Chu. Benchmarking state-of-the-art deep learning software tools. arXiv:1608.07249v7 [cs. DC] 17 Feb 2017. Available at: https://arxiv.org/pdf/1608.07249.pdf (assessed 26 June 2020).

18. Grigorenko E. «Pochemu CNTK?» [«Why CNTK?»]. Available at: https://habr.com/ru/company/microsoft/blog/336552/ (assessed 26 June 2020).

Information about the authors

Ivan V. Evsyutkin, assistant, National Research Tomsk Polytechnic University.

Nikolay G. Markov, Dr. Sc., professor, National Research Tomsk Polytechnic University.
19. "7 Types of Neural Network Activation Functions: How to Choose?». Available at: https://missinglink.ai/guides/neuralnetwork-concepts/7-types-neural-network-activation-functionsright/ (assessed 26 June 2020).

20. Baptista D., Rodrigues S., Morgado-Dias F. Performance comparison of ANN training algorithms for classification Conference: Intelligent Signal Processing (WISP). IEEE $8^{\text {th }}$ International Symposium. Funchal, Portugal, 2013. pp. 115-120.

Received: 3 November 2020. 